LA-UR- $09-00^{2} 753$

Approved for public release,

distribution is unlimited.

Title:

MIMETIC FINITE DIFFERENCE METHOD FOR THE STOKES PROBLEM ON POLYGONAL MESHES

Author(s):

L. BEIRAO DA VEIGA

V. GYRYA

K. LIPNIKOV

G. MANZINI

Intended for:

JOURNAL:

JOURNAL OF COMPUTATIONAL PHYSICS

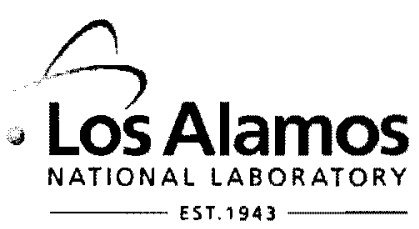

Los Alamos National Laboratory, an affirmative action/equal opportunity employer, is operated by the Los Alamos National Security, LLC for the National Nuclear Security Administration of the U.S. Department of Energy under contract DE-AC52-06NA25396. By acceptance of this article, the publisher recognizes that the U.S. Government retains a nonexclusive, royalty-free license to publish or reproduce the published form of this contribution, or to allow others to do so, for U.S. Government purposes. Los Alamos National Laboratory requests that the publisher identify this article as work performed under the auspices of the U.S. Department of Energy. Los Alamos National Laboratory strongly supports academic freedom and a researcher's right to publish; as an institution, however, the Laboratory does not endorse the viewpoint of a publication or guarantee its technical correctness.

Form $836(7 / 06)$ 


\title{
Mimetic finite difference method for the Stokes problem on polygonal meshes
}

\author{
$\begin{array}{llll}\text { L. Beirão da Veiga } & \text { V. Gyrya } & \text { K. Lipnikov } & \text { G. Manzini } \\ & \$\end{array}$
}

December 11, 2008

\begin{abstract}
Various approaches to extend the finite eleinent methods to non-traditional elements (pyramids, polyhedra, etc.) have been developed over the last decade. Building of basis functions for such elements is a challenging task and may require extensive geometry analysis. The mimetic finite difference (MFD) method has many similarities with low-order finite element methods. Both methods try to preserve fundamental properties of physical and mathematical models. The essential difference is that the MFD method uses only the surface representation of discrete unknowns to build stiffness and mass matrices. Since no extension inside the mesh element is required, practical implementation of the MFD method is simple for polygonal meshes that may include degenerate and non-convex elements. In this article, we develop a MFD method for the Stokes problem on arbitrary polygonal meshes. The method is constructed for tensor coefficients, which will allow to apply it to the linear elasticity problem. The numerical experiments show the second-order convergence for the velocity variable and the first-order for the pressure.
\end{abstract}

\section{Introduction}

Stokes flow is fluid flow where advective inertial forces are negligibly small compared to viscous forces. This is a typical situation on a microscale or when the fuid velocity is very small. Stokes flow is a good and important approximation for a number of physical problems such as sedimentation, modeling of bio-suspensions, construction of efficient fibrous filters and development of energy efficient micro-fluidic devices (e.g. mixers). Efficient numerical solution of Stokes flow requires unstructured meshes adapted to geometry and solution as well as accurate discretization methods capable of treating such meshes. We developed a new mimetic finite difference (MFD) method that remains accurate on general polygonal meshes that may include non-convex and degenerate elements.

General polygonal and polyhedral meshes are often used in complex simulations (see e.g. [18]). In adaptive solution of PDEs on quadrilateral and hexahedral meshes, local refinement of the mesh creates degenerate elements that have $180^{\circ}$ angles. The termination by thinning or tapering out of a geological layer is modeled with degenerate hexahedra that have a few vertices with the same coordinates. Lagrangian meshes may result in non-convex elements. The MFD method provides accurate discretization of PDEs on meshes with all types of degenerate elements.

\footnotetext{
The Pennsylvania State University, Department of Mathematics, University Park, PA 16802, USA E-mail: gyrya@math.psu.edu

${ }^{2}$ Los Alamos National Laboratory, MS B284, Los Alamos, NM 87545, USA E-mail: lipnikovolan.gov

3Dipartimento di Matematica "F.Enriques" Via Saldini 50, 20133 Milano, Italy

E-mail: beirao@mat.unimi.it.

Istituto di Matematica Applicata e Tecnologie Informatiche-CNR, Via Ferrata 1, 27100 Pavia, Italy E-mail: Marco.Manzinioimati.enr,it
} 
The MFD methods $[28,10,12,11,8]$ have many similarities with finite element (FE) methods. Both methods try to preserve fundamental properties of physical and mathematical models such as conservation laws, solution symmetry and positivity, and the fundamental identities and theorems of vector and tensor calculus. Various approaches to extend the FE methods to nontraditional elements (pyramids, polyhedra, etc) have been developed over the last decade (see, e.g. $[20,24,25,30,31])$. Building of basis functions for such elements is a challenging task and may require extensive geometry analysis. For instance, an auxiliary simplicial partition is used in [24]. The MFD methods combine analytical power of FE methods with flexibility provided by polygonal and polyhedral meshes. Contrary to the FE methods, the MFD methods use only surface representation of discrete unknowns to build stiffness and mass matrices. Since no extension inside the mesh element is required, practical implementation of the MFD methods is simple for polygonal and polyhedral meshes.

The MFD methods have been successfully employed for solving diffusion [8, 11, 22], convectiondiffusion [Manzini], electromagnetics [22] and elasticity [2] problems and for modeling fluid flows $[1,13,26]$. The original MFD methods were the low-order methods. Miscellaneous approaches were developed to build higher-order methods $[27,5,21,6]$. In this article, we build a new MFD method for the Stokes problem on polygonal meshes. Derivation of the new method is based on the methodology proposed originally in [12] for diffusion problems. The formulation of the Stokes problem involves a tensor viscosity coefficient so that the developed method may be also used to solve a linear elasticity problem in the displacement formulation. Note that a mixed formulation of the elasticity problem is used in [2].

The developed MFD method is second-order accurate for the fluid velocity and first-order accurate for the pressure. In fact, we build a family of methods with equivalent properties. Detailed analysis of this family will the topic for future research.

A posteriori error estimates is an important part in development of MFD methods. A local error estimator for the diffusion problem is presented, analyzed and tested in [3,4], while in [14] a post-processing methodology is introduced. Finally, we mention a few relevant finite volume discretization methods on polygonal and polyhedral meshes (see $[17,19]$ and references there in). Like the MFD method, these methods are build to preserve important properties of continuum equations.

The paper outline is as follows. In Section 2, we derive the variational form of the Stokes problem. In Section 3, we derive the new MFD method. In Section 4, we analyze the stability of the discretization. In Section 5, we illustrate the proposed method with three numerical experiments.

\section{Variational formulation for the Stokes equation}

Let $\Omega$ be a polygonal domain with the Lipschitz continuous boundary. Let us consider an incompressible Stokes equation

$$
\left\{\begin{aligned}
-2 \operatorname{div}(\nu D(\mathbf{u})) & =\mathbf{F}-\nabla p \\
\operatorname{div}(\mathbf{u}) & =0
\end{aligned} \quad \text { in } \Omega\right.
$$

where $\mathbf{u}$ is the fluid velocity, $p$ is the pressure, $\mathbf{F}$ is a given external force, $\nu$ is the fourth-order symmetric positive definite tensor;

$$
\nu_{k l n m}=\nu_{l k n m}=\nu_{n m k l}=\nu_{m n k l},
$$

and $D(\mathbf{u})$ is the symmetrized gradient,

$$
2 D(\mathbf{u})=\nabla \mathbf{u}+(\nabla \mathbf{u})^{T} .
$$


We impose the Dirichlet boundary conditions on $\Gamma_{1} \subset \partial \Omega$ and the Neumann boundary conditions on $\Gamma_{2}=\partial \Omega \backslash \Gamma_{1}$, where both $\Gamma_{1}$ and $\Gamma_{2}$ are a finite union of connected components. We obtain

$$
\begin{aligned}
\mathbf{u}(\mathbf{x}) & =\mathbf{g}(\mathbf{x}) & & \text { for } \mathbf{x} \in \Gamma_{1}, \\
\sigma(\mathbf{u}(\mathbf{x}), p(\mathbf{x})) \mathbf{n}(\mathbf{x}) & =\mathbf{h}(\mathbf{x}) & & \text { for } \mathbf{x} \in \Gamma_{2}
\end{aligned}
$$

where $\sigma$ is the stress tensor,

$$
\sigma(\mathbf{u}, p)=2 \nu D(\mathbf{u})-p I
$$

Let $X=\left(H^{1}(\Omega)\right)^{2}$. The admissible class of velocity fields $\mathbf{u}$ is defined as

$$
X_{\mathbf{g}}=\left\{\mathbf{u} \in X: \mathbf{u}(\mathbf{x})=\mathbf{g}(\mathbf{x}) \text { for } \mathbf{x} \in \Gamma_{1}\right\}
$$

Note that we do not require functions in $X_{g}$ to be incompressible (incompressibility is enforced by an additional constraint). We will also need a linear space $X_{0}$ of variations in the admissible class $X_{\mathrm{g}}$. It is defined by setting $\mathrm{g}=0$ in (4). Multiplying (1) by $\mathrm{v} \in X_{0}$, integrating by parts over $\Omega$ and using the boundary conditions (3), we get

$$
\int_{\Omega} \nu D(\mathbf{u}): \nabla \mathbf{v} d \mathbf{x}-\int_{\Omega} p \operatorname{div}(\mathbf{v}) d \mathbf{x}=\int_{\Omega} \mathbf{F} \cdot \mathbf{v} d \mathbf{x}+\int_{\Gamma_{2}} \mathbf{h} \cdot \mathbf{v} d \mathbf{x}
$$

Let us introduce the following notations:

$$
\begin{aligned}
\mathcal{A}(\mathbf{u}, \mathbf{v}) & :=\int_{\Omega}(2 \nu D(\mathbf{u})): D \mathbf{v} d \mathbf{x} \\
\mathcal{B}(p, \mathbf{v}) & :=\int_{\Omega} p \operatorname{div}(\mathbf{v}) d \mathbf{x} \\
\mathcal{L}(\mathbf{v}) & :=\int_{\Omega} \mathbf{F} \cdot \mathbf{v} d \mathbf{x}+\int_{\Gamma_{2}} \mathbf{h} \cdot \mathbf{v} d \mathbf{x} .
\end{aligned}
$$

Note that the bilinear form $\mathcal{A}(\mathbf{u}, \mathbf{v})$ is symmetric since $\nabla \mathbf{v}$ can be replaced with $D(\mathbf{v})$. In these notations, equation (5) takes the following form

$$
\mathcal{A}(\mathbf{u}, \mathbf{v})-\mathcal{B}(p, \mathbf{v})=\mathcal{L}(\mathbf{v})
$$

Now we multiply the incompressibility equation in (1) by $q \in L^{2}(\Omega)$ and integrate over the domain $\Omega$ to get a variational formulation: Find a pair $(\mathbf{u}, p), \mathbf{u} \in X_{g}$ and $p \in L^{2}(\Omega)$, such that

$$
\begin{cases}\mathcal{A}(\mathbf{u}, \mathbf{v})-\mathcal{B}(p, \mathbf{v})=\mathcal{L}(\mathbf{v}) & \forall \mathbf{v} \in X_{0} \\ \mathcal{B}(q, \mathbf{u})=0 & \forall q \in L^{2}(\Omega)\end{cases}
$$

In the sequel we assume for simplicity that the measure of $\Gamma_{1}$ is positive, in order to have the uniqueness of the vector variable $\mathbf{u}$. Furthermore note that, in the case $\Gamma_{1}=\partial \Omega$, the pressure variable is defined up to a global constant and the boundary datum must satisfy the consistency condition $\int_{\partial \Omega} \mathbf{g} \cdot \mathbf{n}=0$, with $\mathbf{n}$ the outward unit normal to $\partial \Omega$.

\section{Discretization on polygons}

Let $\Omega^{h}$ be a partition of the computational domain $\Omega$ into $\mathcal{N}\left(\Omega^{h}\right)$ polygons $E$. We assume that this partition is conformal, i.e. intersection of two different elements $E_{1}$ and $E_{2}$ is either a few mesh points, or a few mesh edges (two adjacent elements may share more than one edge) or empty. We allow $\Omega^{h}$ to contain non-convex and degenerate elements.

We assume that the coefficient $\nu$ is a constant tensor inside each mesh element.

The discretization of problem (10) requires to discretize scalar (elements of $L^{2}(\Omega)$ ) and vector (elements of $\left.H^{1}(\Omega)\right)$ functions, the bilinear forms $\mathcal{A}(\mathbf{u}, \mathbf{v})$ and $\mathcal{B}(p, \mathbf{v})$, and the linear form $\mathcal{C}(\mathbf{v})$. 
We begin by introducing degrees of freedom for the functions (details are presented later):

$$
\begin{array}{lll}
p, s \in L^{2}(\Omega) & \rightarrow & P, S \in Q^{h}, \\
\mathbf{u}, \mathbf{v} \in H^{1}(\Omega) & -\rightarrow & U, V \in X^{h} .
\end{array}
$$

We also define subsets of $X^{h}$ approximating $X_{\mathbf{g}}$ and $X_{0}$ to be $X_{\mathbf{g}}^{h}$ and $X_{0}^{h}$, respectively. Next, we discretize the bilinear and linear forms:

$$
\begin{array}{rll}
\mathcal{A}(\mathbf{u}, \mathbf{v}) & -\rightarrow & V^{T} \mathbf{A} U, \\
\mathcal{B}(p, \mathbf{v}) & -\rightarrow & V^{T} \mathbf{D} P \\
\mathcal{L}(\mathbf{v}) & -\rightarrow & V^{T} L,
\end{array}
$$

where $\mathbf{A}$ is symmetric semi-definite matrix with three null models corresponding to the rigid body motion. This gives the following algebraic problem: Find $U \in X_{g}^{h}$ and $P \in Q^{h}$ such that

$$
\begin{aligned}
V^{T} \mathbf{A} U-V^{T} \mathbf{D} P & =V^{T} L & & \forall V \in X_{0}^{h}, \\
U^{T} \mathbf{D} S & =0 & & \forall S \in Q^{h} .
\end{aligned}
$$

This problem can be written in the following matrix form

$$
\left[\begin{array}{l}
V \\
S
\end{array}\right]^{T}\left[\begin{array}{cc}
\mathbf{A} & \mathbf{D}^{T} \\
\mathbf{D} & 0
\end{array}\right]\left[\begin{array}{c}
U \\
P
\end{array}\right]=\left[\begin{array}{c}
L \\
0
\end{array}\right] \quad \forall V \in X_{0}^{h} \text { and } \forall S \in Q^{h}
$$

In practice, it may be convenient to eliminate degrees of freedom corresponding to the Dirichlet boundary conditions (see section 3.5 ). This results in a saddle point problem:

$$
\left[\begin{array}{cc}
\mathbf{A}_{0} & -\mathbf{D}_{0}^{T} \\
-\mathbf{D}_{0} & 0
\end{array}\right]\left[\begin{array}{c}
U_{0} \\
P
\end{array}\right]=\left[\begin{array}{l}
G_{U} \\
G_{P}
\end{array}\right]
$$

where $\mathbf{A}_{0}$ and $\mathbf{D}_{0}$ are sub-matrices of $\mathbf{A}$ and $\mathbf{D}$, respectively. Now the matrix $\mathbf{A}_{0}$ is symmetric positive definite and a number of iterative solvers can be used to solve the saddle point problem (14).

\subsection{Discretization of scalar and vector functions}

Let us consider a sample element $E$, shown on Fig.1. We denote the number of its vertices by $\mathcal{N}(E)$. Note that the number of its edges is also $\mathcal{N}(E)$. Let $\mathbf{n}_{E}(\mathbf{x})$ be the external unit normal vector to the boundary $\partial E$ at the point $\mathbf{x}$.

For scalar functions (e.g., pressure $p$ ), we specify one degree of freedom per element, $p_{E}$, for instance, the value of $p$ at the center of mass of $E$. The local approximation space $Q_{E}$ has dimension one and isomorphic to the space of constant functions on $E$. Dimension of the global space $Q^{h}$ equals to the number of mesh elements, $\mathcal{N}\left(\Omega^{h}\right)$.

Let us specify degrees of freedom for vector functions such as the velocity $u$. For each vertex $\mathbf{a}_{i}$ of a polygonal element $E$, we define two degrees of freedom: the value of $\mathbf{u}$ at $\mathbf{a}_{i}$, i.e.

$$
\left(U_{i}^{x}, U_{i}^{y}\right)^{T}:=\mathbf{u}\left(\mathbf{a}_{i}\right), \quad i=1, \ldots, \mathcal{N}(E) .
$$

For each edge $e_{i}$ of $E$, we specify one degree of freedom: the average flux through the edge, i.e.

$$
U_{i}^{e}:=\frac{1}{\left|e_{i}\right|} \int_{e_{i}} \mathbf{u}(s) \cdot \mathbf{n}_{E} d s .
$$

The local approximation space $X_{E}^{h}$ on the polygonal element $E$ is uniquely characterized by $3 \mathcal{N}(E)$ degrees of freedom. The dimension of the global space $X^{h}$ is twice the number of mesh 

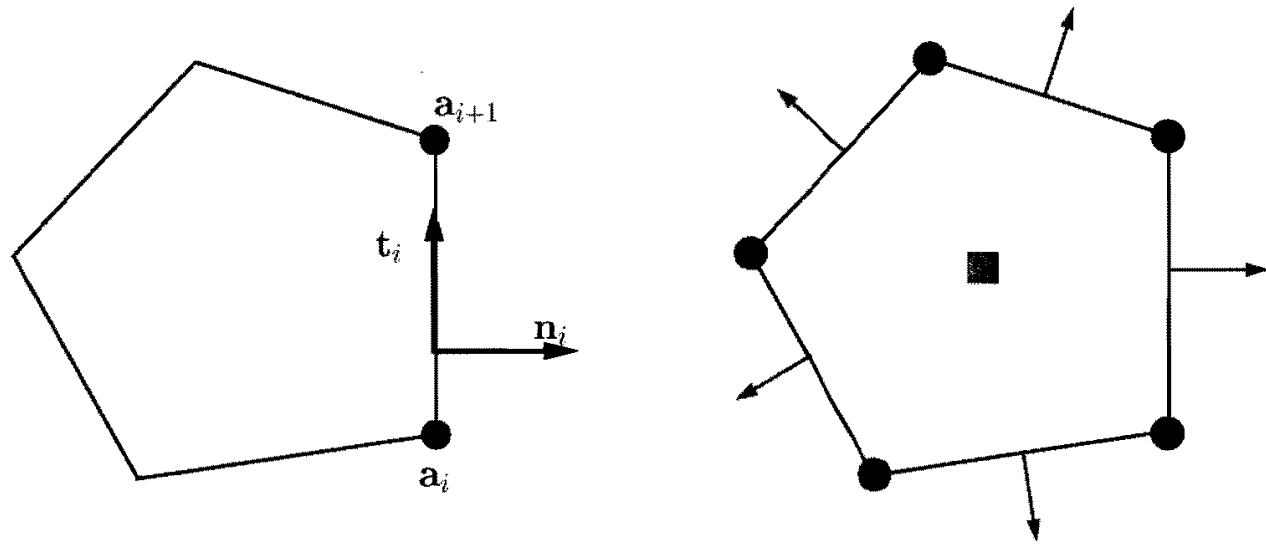

Figure 1: Left picture introduces notations used through the paper. Right picture shows the degrees of freedom for the pressure (red box) and velocity (blue circles and arrows).

vertices plus the number of mesh edges. The dimension of spaces $X_{3}^{h}$ and $X_{0}^{h}$ equals to the dimension of $X^{h}$ minus twice the number of Dirichlet points and the number of Dirichlet edges.

Let $U_{E}$ be the restriction of $U \in X^{h}$ to the element $E$,

$$
U_{E}=\left(U_{1}^{x}, U_{1}^{y}, U_{1}^{e}, U_{2}^{x}, U_{2}^{y}, U_{2}^{e}, \ldots, U_{\mathcal{N}(E)}^{x}, U_{\mathcal{N}(E)}^{y}, U_{\mathcal{N}(E)}^{e}\right)^{T} .
$$

Later we show that the space $X_{E}$ of algebraic vectors of size $\mathcal{D}(E) \equiv 3 \mathcal{N}(E)$ is isomorphic to a specially designed space $\mathcal{V}_{E}$ of vector functions. In other words, for every $U_{E}$ in $X_{E}$, there exists a unique vector function $\mathbf{u}_{E}$ in $\mathcal{V}_{E}$.

\subsection{Discretization of $\mathcal{A}(\mathrm{u}, \mathrm{v})$}

Let us break the bilinear form $\mathcal{A}(\mathbf{u}, \mathbf{v})$ into smaller pieces:

$$
\mathcal{A}(\mathbf{u}, \mathbf{v})=\sum_{E \in \Omega_{h}} \mathcal{A}_{E}\left(\mathbf{u}_{E}, \mathbf{v}_{E}\right)
$$

which is the standard step in assembling a stiffness matrix. Let $U_{E}, V_{E} \in X_{E}$ be vector representations of functions $\mathbf{u}_{E}, \mathbf{v}_{E} \in \mathcal{V}_{E}$. Then,

$$
U_{E}^{T} \mathbf{A}_{E} V_{E} \equiv \mathcal{A}_{E}\left(\mathbf{u}_{E}, \mathbf{v}_{E}\right)=\int_{E} 2 \nu D\left(\mathbf{u}_{E}\right): D\left(\mathbf{v}_{E}\right) d \mathbf{x} .
$$

The goal of this section is to derive formulas for calculating entries of elemental matrices $\mathbf{A}_{E}$. So far, we have followed roughly the finite element path. The next step would require to formulate basis functions for a polygonal element which is a challenging problem. From this moment and

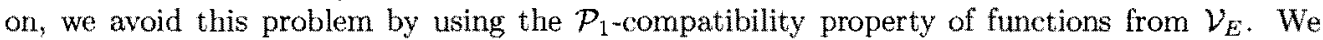
write $\mathcal{V}_{E}$ as a direct sum of two linear spaces:

$$
\nu_{E}=\nu_{E, 1} \oplus \mathcal{V}_{E, 2}
$$

We define $\mathcal{V}_{E, 1}$ as a space of linear vector functions, $\mathcal{V}_{E, 1}=\left(\mathcal{P}_{1}(E)\right)^{2}$. The dimension of $\mathcal{V}_{E, 1}$ is six. The linear part in $\mathcal{V}_{E}$ is necessary to have a second-order convergence method. The remaining part is defined only partially. The following is the direct consequence of the integration by parts formula. 


\subsection{1 $\mathcal{P}_{1}$-compatibility property}

Let $\mathbf{u}_{1} \in V_{E, 1}$ and $\nu$ be a constant tensor. Then, the $\mathcal{P}_{1}$-compatibility property states that

$$
\int_{E} \nu D\left(\mathbf{u}_{1}\right): D\left(\mathbf{v}_{E}\right) d \mathbf{x}=\int_{\partial E}\left(\nu D\left(\mathbf{u}_{1}\right) \cdot \mathbf{n}_{E}\right) \cdot \mathbf{v}_{E} d s \quad \forall \mathbf{v}_{E} \in \mathcal{V}_{E}
$$

This implies that calculation of a part of the stiffness matrix $\mathbf{A}_{E}$ requires to know $\mathbf{v}_{E}$ only on edges of $E$. It is sufficient to build a convergent scheme. We make two assumptions.

(A1) For any $\mathbf{v}_{E} \in \mathcal{V}_{E}$, its normal component $\mathbf{v}_{E} \cdot \mathbf{n}_{E}$ is quadratic on every edge $e_{i}$ of $E$. Thus, it is uniquely determined by the flux $V_{i}^{e}$ and four degrees of freedom at the vertices $\mathbf{a}_{i}$ and $\mathbf{a}_{i+1}$.

(A2) For any $\mathbf{v}_{E} \in \mathcal{V}_{E}$, its tangential component is linear on every edge $e_{i}$ of $E$. Thus, it is uniquely determined by four degrees of freedom at the vertices $\mathbf{a}_{i}$ and $\mathbf{a}_{i+1}$.

These conditions imply continuity of a discrete velocity function across mesh edges. Using notation on Fig. 1 and the second condition, we immediately have

$$
\int_{e_{i}} \mathbf{v}_{E} \cdot \mathbf{t}_{i} d s=\frac{\left|e_{i}\right|}{2}\left(V_{i}^{x}, V_{i}^{y}\right)^{T} \cdot \mathbf{t}_{i}+\frac{\left|e_{i}\right|}{2}\left(V_{i+1}^{x}, V_{i+1}^{y}\right)^{T} \cdot \mathbf{t}_{i},
$$

where $\mathbf{t}_{i}$ is a tangential unit vector to $e_{i}$.

Let $\phi_{i}, i=1, \ldots, \mathcal{D}(E)$ form a basis in $\mathcal{V}_{E}$. The first six vector functions forming a basis in $\mathcal{V}_{E, 1}$ are

$$
\begin{aligned}
& \phi_{1}(x, y)=\left[\begin{array}{l}
1 \\
0
\end{array}\right], \quad \phi_{2}(x, y)=\frac{1}{2}\left[\begin{array}{c}
y \\
-x
\end{array}\right], \quad \phi_{3}(x, y)=\left[\begin{array}{l}
0 \\
1
\end{array}\right], \\
& \phi_{4}(x, y)=\left[\begin{array}{l}
x \\
0
\end{array}\right], \quad \phi_{5}(x, y)=\frac{1}{2}\left[\begin{array}{l}
y \\
x
\end{array}\right], \quad \phi_{6}(x, y)=\left[\begin{array}{l}
0 \\
y
\end{array}\right] .
\end{aligned}
$$

Note that the first three basis functions span the space of the rigid body motions, while $\phi_{1}$ and $\phi_{3}$ alone span the space of translations. The final restriction on the remaining basis functions $\phi_{j}$ (forming the basis in $\mathcal{V}_{E, 2}$ ) is

$$
\mathcal{A}_{E}\left(\phi_{i}, \phi_{j}\right)=0, \quad 1 \leq i \leq 6<j \leq \mathcal{D}(E) .
$$

As shown later, this assumption simplifies the structure of the matrix $\mathbf{A}_{E}$.

\subsubsection{Change of basis in $X_{E}$}

Let transformation matrix $\mathbf{T}_{E}$ act from the basis defined by functions $\phi_{i}$ to the basis defined by the degrees of freedom (the natural basis in $X_{E}$ ),

$$
\mathbf{T}_{E}=\left\{T_{E, i, j}\right\}, \quad T_{E, i, j}-i \text {-th degree of freedom of } \phi_{j} .
$$

The first six column of $\mathbf{T}_{E}$ are uniquely defined by the choice of the basis functions (19). The remaining columns $T_{j}, j>6$, are defined using (20) and the $\mathcal{P}_{1}$-compatibility property. Let $R\left(\phi_{j}\right), j=1,2, . .6$, indicate the computable vector in $\mathbb{R}^{\mathcal{D}}$ which represents the right hand side of $(17)$

$$
R\left(\phi_{j}\right)^{T} V_{E}=\int_{\partial E}\left(\nu D\left(\phi_{j}\right) \cdot \mathbf{n}_{E}\right) \cdot \mathbf{v}_{E} d s \quad \forall V_{E} \in X_{E} \equiv \mathbb{R}^{\mathcal{D}(E)}
$$

Then, taking $\mathbf{u}_{1}=\phi_{i}$ and $\mathbf{v}_{E}=\phi_{j}$ in (17), we get

$$
T_{i}^{T} \mathbf{A}_{E} T_{j}=R\left(\phi_{i}\right)^{T} T_{j}, \quad 1 \leq i \leq 6<j \leq \mathcal{D}(E) .
$$


The first three basis functions $\phi_{i}(i=1,2,3)$ in $\mathcal{V}_{E}$ correspond to the rigid body motions. Therefore, $R\left(\phi_{1}\right)=R\left(\phi_{2}\right)=R\left(\phi_{3}\right)=0$ and we get only three conditions to define $\mathcal{D}(E)-6$ basis vectors in $X_{E}$. We add three additional conditions

$$
T_{i}^{T} T_{j}=0 \quad 1 \leq i \leq 3<6<j \leq \mathcal{D}(E)
$$

Let $\mathbf{T}_{i-j}$ be the $\mathcal{D}(E) \times(j-i+1)$ matrix obtained by selecting the consecutive columns of $\mathbf{T}_{E}:$

$$
\mathbf{T}_{i-j}=\left[T_{i}, T_{i+1}, \ldots, T_{j}\right] .
$$

Using this notation, we may write $\mathbf{T}_{1-\mathcal{D}}$ instead of $\mathbf{T}_{E}$. Hereafter, to simplify notations, we shall write $\mathcal{D}$ instead of $\mathcal{D}(E)$. We introduce similar notations for matrices $\mathbf{R}_{i-j}$ formed by vectors $R_{i}=R\left(\phi_{i}\right), i=1, \ldots, 6$. Let us define $\mathbf{R}_{E} \equiv \mathbf{R}_{1-6}$ and summarize the above results:

$$
\begin{aligned}
& \mathbf{R}_{1-3}=\mathbf{0} \\
& \mathbf{A}_{E} \mathbf{T}_{1-6}=\mathbf{R}_{E} \\
& \mathbf{T}_{1-6}^{T} \mathbf{R}_{E}=\mathbf{T}_{1-6}^{T} \mathbf{A}_{E} \mathbf{T}_{1-6}=: \tilde{\mathbf{A}}_{E}^{11} \\
& \mathbf{T}_{7-\mathcal{D}}^{T} \mathbf{A}_{E} \mathbf{T}_{1-6}=\mathbf{T}_{7-\mathcal{D}}^{T} \mathbf{R}_{E}=\mathbf{0} \\
& \mathbf{T}_{7-\mathcal{D}}^{T} \mathbf{T}_{\mathbf{1 - 3}}=\mathbf{0}
\end{aligned}
$$

Note that the entries of the symmetric $6 \times 6$ matrix $\tilde{\mathbf{A}}_{E}^{11}$ defined in (26) are calculated directly using the basis functions (19) in formula (17). Since these basis functions are linear, the integration is simple. Moreover, (24) implies that the corresponding entries of matrix $\tilde{\mathbf{A}}_{E}^{11}$ are zeros,

$$
\tilde{\mathbf{A}}_{E}^{11}=\left[\begin{array}{cc}
0 & 0 \\
0 & \tilde{\mathbf{S}}_{E}^{11}
\end{array}\right],
$$

where each block is a $3 \times 3$ matrix. In a special case when $\nu$ is a scalar, the matrix $\tilde{\mathbf{S}}_{E}^{11}$ is diagonal. In a general case, this matrix is positive definite for any positive definite tensor $\nu$.

Observe that the last $\mathcal{D}-6$ columns of $\mathbf{T}_{E}$ can be chosen to be mutually orthogonal and can be scaled arbitrarily. It will be convenient to assume that

$$
\mathbf{T}_{7-\mathcal{D}}^{T} \mathbf{T}_{7-\mathcal{D}}=|E| \mathbf{I}_{\mathcal{D}-6}
$$

where $\mathbf{I}_{\mathcal{D}-6}$ represents the identity matrix.

The following results shows that vectors $T_{j}, j=1, \ldots, \mathcal{D}$, do form a basis in $X_{E}$.

Lemma 1. The matrix $\mathrm{T}_{E} \in \mathbb{R}^{\mathcal{D} \times \mathcal{D}}$ is invertible.

Proof. The proof is by contradiction. Let us assume that $\mathbf{T}_{E} V=0$. Using (25)-(27), we easily obtain

$$
0=\mathbf{A}_{E}^{T} \mathbf{T}_{E} V=\tilde{\mathbf{A}}_{E}^{11}\left[V_{1}, V_{2}, \ldots, V_{6}\right]^{T}=\tilde{\mathbf{S}}_{E}^{11}\left[V_{4}, V_{5}, V_{6}\right]
$$

Since $\mathbf{S}_{E}^{11}$ is positive definite, the above identity gives $V_{4}=V_{5}=V_{6}=0$. Due to the definition of $T_{1}, T_{2}, T_{3}$ and the orthogonality relations (28), (30), the columns $T_{j}$ with $j=1,2,3,7,8, ., \mathcal{D}$ are linearly independent. Therefore their linear combination is zero only then the corresponding weights are zeros. Thus, $V=0$. This proves the assertion of the lemma.

Let us define the representation of matrix $\mathbf{A}_{E}$ in the new basis $T_{1}, \ldots, T_{D}$ :

$$
\tilde{\mathbf{A}}_{E}=\mathbf{T}_{E}^{T} \mathbf{A}_{E} \mathbf{T}_{E}
$$


The orthogonality (20) and property (26) imply the following $2 \times 2$ partition of $\tilde{\mathbf{A}}_{E}$ :

$$
\tilde{\mathbf{A}}_{E}=\left[\begin{array}{cc}
\tilde{\mathbf{A}}_{E}^{11} & 0 \\
0 & \tilde{\mathbf{A}}_{E}^{22}
\end{array}\right] \text {. }
$$

The matrix $\tilde{\mathbf{A}}_{E}^{22}$ can be arbitrary positive definite matrix whose eigenvalues are close to the maximum eigenvalue of $\tilde{\mathbf{A}}_{E}^{11}$. Various choices of $\tilde{\mathbf{A}}_{E}^{22}$ define a family of numerical methods with equivalent approximation properties. In practice, a reasonable choice for $\tilde{\mathbf{A}}_{E}^{22}$ is the scalar matrix,

$$
\tilde{\mathbf{A}}_{E}^{22}=\lambda_{\max }(\nu)|E| \mathbf{I}_{\mathcal{D}-6}
$$

This completes construction of the matrix $\mathbf{A}_{E}$.

Assumptions $\mathbf{A} 1$ and $\mathbf{A} 2$ give unique description of function $\mathbf{v}_{E}$ on every edge of $E$. Let us illustrate how to compute the integral over edge $e_{i}$ in the right-hand side of (17). Since $\nu$ is a constant tensor, $\mathbf{u}_{1}$ is a linear vector function, we get that $\mathbf{c} \equiv 2 \nu D\left(\mathbf{u}_{1}\right) \cdot \mathbf{n}_{i}$ is a constant vector function and

$$
\begin{aligned}
\int_{e_{i}}\left(2 \nu D\left(\mathbf{u}_{1}\right) \cdot \mathbf{n}_{i}\right) \cdot \mathbf{v}_{E} d s & =\int_{e_{i}}\left(\mathbf{c} \cdot \mathbf{n}_{i}\right)\left(\mathbf{v} \cdot \mathbf{n}_{i}\right) d s+\int_{e_{i}}\left(\mathbf{c} \cdot \mathbf{t}_{i}\right)\left(\mathbf{v} \cdot \mathbf{t}_{i}\right) d s \\
& =\left(\mathbf{c} \cdot \mathbf{n}_{i}\right)\left|e_{i}\right| V_{i}^{e}+\left(\mathbf{c} \cdot \mathbf{t}_{i}\right) \frac{\left|e_{i}\right|}{2}\left(V_{i}^{x}+V_{i+1}^{x}, V_{i}^{y}+V_{i+1}^{y}\right)^{T} \cdot \mathbf{t}_{i}
\end{aligned}
$$

\subsubsection{Inexpensive construction of the stiffness matrix $\mathbf{A}_{E}$}

Calculation of the matrix $\mathbf{A}_{E}$ described above involves inversion of the transformation matrix $\mathbf{T}_{E}$. For a quadrilateral element it is a $12 \times 12$ matrix. In general, complexity of the above procedure must be compared with the complexity of iterative solvers for the saddle point problem (14) that can be quite a challenge. However, a slight modification of the above argument gives a cheaper method for calculating $\mathbf{A}_{E}$.

Property (26) implies that

$$
\mathbf{R}_{4-6}^{T} \mathbf{T}_{4-6}=\tilde{\mathbf{S}}_{E}^{11}
$$

Then, the general form of the matrix $\mathbf{A}_{E}$ is

$$
\mathbf{A}_{E}=\mathbf{R}_{4-6}\left(\tilde{\mathbf{S}}_{E}^{11}\right)^{-1} \mathbf{R}_{4-6}^{T}+\mathbf{P} \mathbf{U}_{E} \mathbf{P}
$$

where $\mathbf{P}$ is the orthogonal projector,

$$
\mathbf{P}=\mathbf{I}_{\mathcal{D}}-\mathbf{T}_{1-6}\left(\mathbf{T}_{1-6}^{T} \mathbf{T}_{1-6}\right)^{-1} \mathbf{T}_{1-6}^{T},
$$

and $\mathbf{U}_{E}$ is an arbitrary symmetric positive definite matrix. This new matrix $\mathbf{A}_{E}$ belongs to the same family of matrices given by (33) and (32). However, now we need to invert only the $3 \times 3$ matrix $\tilde{\mathbf{S}}_{E}^{11}$ and the $6 \times 6$ matrix $\mathbf{T}_{1-6}^{T} \mathbf{T}_{1-6}$.

For a scalar coefficient $\nu$, the matrix $\tilde{\mathbf{S}}_{E}^{11}$ has a very simple form, $\tilde{\mathbf{S}}_{E}^{11}=2 \nu|E| \mathbf{I}_{3}$. If we choose $\mathrm{U}_{E}$ to be a scalar matrix, formula $(35)$ is simplified:

$$
\mathbf{A}_{E}=\frac{2 \nu}{|E|} \mathbf{R}_{4-6} \mathbf{R}_{4-6}^{T}+2 \nu|E| \mathbf{P} \text {. }
$$

The structure of the $6 \times 6$ matrix $\mathbf{T}_{1-6}^{T} \mathbf{T}_{1-6}$ can be further simplified by shifting the Cartesian coordinate system to the center of mass of $E$. In this case, vectors $T_{1}$ and $T_{3}$ (corresponding to $\phi_{1}$ and $\phi_{3}$, respectively) become orthogonal to the remaining vectors. After rearranging columns and rows of $\mathbf{T}_{1-6}^{T} \mathbf{T}_{1-6}$, we get a block-diagonal matrix.

Remark 1. It is possible to find an explicit form for a particular choice of the basis functions $\phi_{i}$ by solving a local problem on $E$ following the path described in [11] for the diffusion problem. Such calculation of basis functions is expensive and therefore is not practical. 


\subsection{Discretization of $\mathcal{B}(p, \mathbf{v})$}

Similar to the previous section, we break the bilinear form $\mathcal{B}(p, \mathbf{v})$ into smaller pieces:

$$
\mathcal{B}(p, \mathbf{v})=\sum_{E \in \Omega^{\prime 4}} \mathcal{B}_{E}\left(p_{E}, \mathbf{v}_{E}\right)
$$

Since the pressure $p_{E}$ is constant on each polygonal element $E$, using the divergence theorem we have

$$
\begin{aligned}
\mathcal{B}_{E}\left(p_{E}, \mathbf{v}_{E}\right) & \equiv \int_{E} p_{E} \operatorname{div}\left(\mathbf{v}_{E}\right) d \mathbf{x}=p_{E} \int_{E} \operatorname{div}\left(\mathbf{v}_{E}\right) d \mathbf{x}=p_{E} \int_{\partial E} \mathbf{v}_{E} \cdot \mathbf{n}_{E} d s \\
& =p_{E} \sum_{e_{i} \in \partial E} \int_{e_{\xi}} \mathbf{v}_{E} \cdot \mathbf{n}_{E} d s=p_{E} \sum_{e_{i} \in \partial E}\left|e_{i}\right| V_{i}^{e}
\end{aligned}
$$

This is a sum of the fluxes through the edges of $E$, which are our degrees of freedom.

\subsection{Discretization of $\mathcal{L}(v)$}

We rewrite $\mathcal{L}(\mathbf{v})$ as follows:

$$
\mathcal{L}(\mathbf{v})=\sum_{E \in \Omega^{h}} \int_{E} \mathbf{F} \cdot \mathbf{v} d \mathbf{x}+\sum_{e \in \Gamma_{2}} \int_{e} \mathbf{h} \cdot \mathbf{v} d s
$$

The edge integral is discretized by assuming that $\mathbf{h}(\mathbf{x})$ is constant on each edge. Let $e_{i}$ be the $i$-th edge of polygonal element $E$ and let $\mathbf{x}_{i}$ be the mid-point of $e_{i}$. Then, using notations in Fig. 1 and assumptions $\mathbf{A 1}$ and $\mathbf{A 2}$, we get

$$
\begin{aligned}
\int_{e_{i}} \mathbf{h} \cdot \mathbf{v}_{E} d s & \approx \mathbf{h}\left(\mathbf{x}_{i}\right) \cdot \int_{e_{i}} \mathbf{v}_{E} d s \\
& =\left(\mathbf{h}\left(\mathbf{x}_{i}\right) \cdot \mathbf{n}_{i}\right) \int_{e_{i}} \mathbf{v}_{E} \cdot \mathbf{n}_{i} d s+\left(\mathbf{h}\left(\mathbf{x}_{i}\right) \cdot \mathbf{t}_{i}\right) \int_{\epsilon_{i}} \mathbf{v}_{E} \cdot \mathbf{t}_{i} d s \\
& =\left(\mathbf{h}\left(\mathbf{x}_{i}\right) \cdot \mathbf{n}_{i}\right)\left|e_{i}\right| V_{i}^{3}+\left(\mathbf{h}\left(\mathbf{x}_{i}\right) \cdot \mathbf{t}_{i}\right) \frac{\left|e_{i}\right|}{2}\left(V_{i}^{1}+V_{i+1}^{1}, V_{i}^{2}+V_{i+1}^{2}\right)^{T} \cdot \mathbf{t}_{i} .
\end{aligned}
$$

The volume integral in (36) is discretized by assuming that $\mathbf{F}(\mathbf{x})$ is constant over each element $E$. Let $\mathbf{x}_{E}$ be the center of mass of $E$. We define a quadrature rule with the quadrature points at vertices of $E$ and positive weights $w_{i}, i=1, \ldots, \mathcal{N}(E)$ such that the quadrature is exact for linear functions. Using this quadrature, we get

$$
\int_{E} \mathbf{F} \cdot \mathbf{v}_{E} d \mathbf{x} \approx \sum_{i=1}^{\mathcal{N}(E)} \omega_{i} \mathbf{F}\left(\mathbf{x}_{E}\right) \cdot \mathbf{v}_{E}\left(\mathbf{a}_{i}\right)=\sum_{i=1}^{\mathcal{N}(E)} \omega_{i}\left(V_{i}^{1}, V_{i}^{2}\right)^{T} \cdot \mathbf{F}\left(\mathbf{x}_{E}\right)
$$

We choose the weights $\omega_{i}$ as the coefficients in a formula that defines the center of mass of $E$ via coordinates of its vertices.

\subsection{Boundary conditions}

In the variational problem, the Dirichlet type boundary conditions manifest themselves through the admissible class (4), while the Neumann type boundary conditions only effect the linear functional $\mathcal{L}(\mathbf{v})$.

The discrete Dirichlet boundary conditions on $\Gamma_{1}$ appear through the definition of the class $X_{g}^{h}$ and the space $X_{0}^{h}$. That is the values of the degrees of freedom corresponding to the vertices and edges on the boundary $\Gamma_{1}$ are prescribed. For a boundary vertex $\mathbf{a}_{i} \in \Gamma_{1}$ we define

$$
\left(U_{i}^{x}, U_{i}^{y}\right)^{T}:=\mathbf{g}\left(\mathbf{a}_{i}\right)
$$


For a boundary edge $e_{i} \in \Gamma_{1}$ we define

$$
U_{i}^{e}:=\frac{1}{\left|e_{i}\right|} \int_{e_{i}} \mathbf{g}(s) \cdot \mathbf{n}_{E} d s .
$$

Let us denote by $U_{0}$ and $V_{0}$ the part of $U$ and $V$, respectively, that does not contain the degrees of freedom specified by the Dirichlet boundary conditions (39)-(40). Substituting (39)(40) into (13) and eliminating equations corresponding to the Dirichlet degrees of freedom, we obtain the linear system (14) for $U_{0}$ and $P$.

\section{Stability analysis}

In this section, we study stability of the MFD discretization, which is important for demonstrating convergence of the numerical method. The stability analysis of saddle point problems [9] requires to prove two inequalities. The first inequality is the coercivity of the bilinear form $\mathcal{A}$ with respect to a natural norm in $X^{h}$ (see Theorem 1). The second inequality is the inf-sup condition (see section 4.2 ).

\subsection{Coercivity of bilinear form $\mathcal{A}$}

\subsubsection{Natural norm in $X_{0}^{h}$}

In order to show the coercivity of the bilinear form $\mathcal{A}$ we need to define a norm on the space $X_{0}^{h}$. Since the shape functions $\phi_{7}, \ldots, \phi_{3 N}$ are not know inside the elements we cannot use $H^{1}$-norm for the space $X_{0}^{h}$. Our goal is to define an analog of $H^{1}$-norm that would only use the known information about the space $X_{0}^{h}$.

Due to (A1) and (A2), for any $V_{E} \in X_{E}$, the corresponding vector function $\mathbf{v}_{E} \in \mathcal{V}_{E}$ is completely determined on $\partial E$. Analogous to $H^{1}$-norm, our norm should depend on the derivative of the function and scale appropriately under change of coordinates/scaling.

Therefore we can define the following seminorm on $X_{E}$ :

$$
\left\|V_{E}\right\|_{E}^{2}:=\sum_{i=1}^{\mathcal{N}(E)}\left|e_{i}\right| \int_{e_{t}}\left(\frac{d}{d s} \mathbf{v}_{E}(s)\right)^{2} d s \quad \forall V_{E} \in X_{E},
$$

where $\frac{d i}{d s}$ is the tangential derivative.

Recall that the basis functions $\phi_{1}, \phi_{2}, \phi_{3}$ represent the rigid motions of $E$ and correspond to the basis vectors $T_{1}, T_{2}, T_{3} \in X_{E}$. The natural local seminorm that vanishes on rigid body motions is

$$
\left\|V_{E}\right\|_{\star, E}:=\min _{\varepsilon \in \mathbb{R}^{3}}\left\|V_{E}+\mathbf{T}_{\mathbf{l}-3 c}\right\|_{E} \quad \forall V_{E} \in X_{E} .
$$

In the above definition, we could ignore basis functions $\phi_{1}$ and $\phi_{3}$ as (41), due to differentiation ignores constant functions.

In the sequel we use $\|\cdot\|$ for the standard Euclidean norm on $\mathbb{R}^{m}$. Whenever $m$ is different from $\mathcal{D}$, we use the lower-case letters $\underline{v}, \underline{u}$ and $\underline{w}$ for vectors in $\mathbb{R}^{m}$. We also introduce the following seminorm:

$$
\|\underline{v}\|_{*}^{2}:=\sum_{i=4}^{m} v_{i}^{2}, \quad \forall \underline{v} \in \mathbb{R}^{m}, \quad m>3 .
$$

Finally, we define the broken seminorm on the space $X^{h}$;

$$
\|V\|_{*}^{2}:=\sum_{E \in \Omega^{h^{*}}}\left\|\mid V_{E}\right\|_{*, E}^{2} .
$$

This seminorm becomes a norm on the space $X_{0}^{h}$. The goal of the next subsections is to prove the coercivity of the bilinear form $\mathcal{A}$ with respect to the norm $\|\cdot\|_{*}$. 


\subsubsection{Mesh regularity assumptions}

A few quite general mesh assumptions introduced in [8] are required for analysis. We assume that there exists a compatible decomposition $S_{h}$ of the polygonal mesh $\Omega_{h}$ into triangles. Moreover, there exist mesh independent numbers $N_{*} \in \mathbb{N}$ and $\rho_{*}>0$, such that:

- every polygon $E \in \Omega_{h}$ admits a decomposition $\left.S_{h}\right|_{E}$ made of less than $N_{*}$ triangles;

- for each triangle $T \in S_{h}$, the ratio of the radius of the inscribed disk to the diameter of $T$ is bounded from below by $\rho_{*}$.

We do not need to build the decomposition $S_{h}$ explicitly. It suffices to know that it does exist. The consequences of the above mesh assumptions are:

- The number of edges $\mathcal{N}(E)$ of each polygon $E$ is uniformly bounded.

- There exists a constant $\sigma_{*}$ dependent only of $N_{*}$ and $\rho_{*}$, such that

$$
\left|e_{i}\right| \geq \sigma_{*} \operatorname{diam}(E) \quad \text { and } \quad|E| \geq \sigma_{*} \operatorname{diam}(E)^{2}
$$

for every edge $e_{i}$ of $E_{i}$.

\subsubsection{Main result}

Hereafter, $C, C_{1}$ and $C_{2}$ are generic positive constants, possibly different at each occurrence, independent of the mesh. These constants may depend on the tensor $\nu$ and the shape regularity parameters $N_{*} i, \rho_{*}$ and $\sigma_{*}$, introduced in Section 4.1.2.

Lemma 2. The symmetric $3 \times 3$ matrix $\tilde{\mathbf{S}}_{E}^{11}$, introduced in $(29)$, satisfies

$$
C_{1}\left|E\|\| \underline{v}\left\|^{2} \leq \underline{v}^{T} \tilde{\mathbf{S}}_{E}^{11} \underline{v} \leq C_{2}|E|\right\| \underline{v}\right|^{2} \quad \forall \underline{v} \in \mathbb{R}^{3} .
$$

Proof. The result follows from the definition of $\tilde{\mathbf{S}}_{E^{*}}^{11}$, noting that $D\left(\phi_{4}\right), D\left(\phi_{5}\right), D\left(\phi_{6}\right)$ are all constant tensors. With a scaling argument

$$
\left(\tilde{\mathbf{S}}_{E}^{11}\right)_{(i-3)(j-3)}=\int_{E} \nu D\left(\phi_{i}\right): D\left(\phi_{j}\right) d \mathbf{x}=|E|\left(\nu D\left(\phi_{i}\right): D\left(\phi_{j}\right)\right) \quad i, j=4,5,6
$$

and that the fourth order tensor $\nu$ is positive definite.

As a consequence of the scaling choice (34) we also have

$$
C_{1}\left|E\|\| \underline{v}\left\|^{2} \leq \underline{v}^{T} \tilde{\mathbf{A}}_{E}^{22} \underline{v} \leq C_{2}|E|\right\| \underline{v} \|^{2} \quad \forall \underline{v} \in \mathbb{R}^{D-6} .\right.
$$

By definition, first using (32)-(33) and then (29) we have

$$
\begin{aligned}
V_{E}^{T} \mathbf{A}_{E} V_{E} & =\underline{w}^{T} \tilde{\mathbf{A}}_{E} \underline{w}=\underline{u}^{T} \tilde{\mathbf{A}}_{E}^{11} \underline{u}+\underline{v}^{T} \tilde{\mathbf{A}}_{E}^{22} \underline{v} \\
& =\left(u_{4}, u_{5}, u_{6}\right) \tilde{\mathbf{S}}_{E}^{11}\left(u_{4}, u_{5}, u_{6}\right)^{T}+\underline{v}^{T} \tilde{\mathbf{A}}_{E}^{22} \underline{v}
\end{aligned}
$$

From this equality, using Lemma 2 and (45) it immediately follows

$$
C_{1}|E|\left(\|\underline{u}\|_{*}^{2}+\|\underline{v}\|^{2}\right) \leq V_{E}^{T} \mathbf{A}_{E} V_{E} \leq C_{2}|E|\left(\|\underline{u}\|_{\star}^{2}+\|\underline{u}\|^{2}\right) .
$$

for all $V_{E} \in X_{E}, \underline{u} \in \mathbb{R}^{6}$ and $\underline{v} \in \mathbb{R}^{\mathcal{D}-6}$ such that

$$
V_{E}=\mathbf{T} \underline{w}=\mathbf{T}_{1-6} \underline{u}+\mathbf{T}_{7-\mathcal{D}} \underline{v} .
$$

We can now present the following Lernma. 
Lemma 3. There exists a positive constant $\alpha_{E}$, depending only on the tensor $\nu$ and the geometric constants in Section 4.1.2, such that

$$
\mathcal{A}_{E}\left(\mathbf{v}_{E}, \mathbf{v}_{E}\right)=V_{E}^{T} \mathbf{A}_{E} V_{E} \geq \alpha_{E}\left\|V_{E}\right\|_{*, E}^{2} \quad \forall V_{E} \in X_{E} .
$$

Proof. Let $V_{E} \in X_{E} \equiv \mathbb{R}^{\mathcal{D}}$. Since the matrix $\mathbf{T}$ is invertible, for each $V_{E}$, there exist two unique vectors $\underline{u} \in \mathbb{R}^{6}$ and $\underline{v} \in \mathbb{R}^{\mathcal{D}-6}$ such that (47) holds.

The triangle inequality gives

$$
\left\|V_{E}\right\|_{*, E}^{2} \leq 2\left\|\mathbf{T}_{1-6} \underline{u}\right\|\left\|_{\star, E}^{2}+2\right\| \mathbf{T}_{7-\mathcal{D}} \underline{v} \|_{\star, E}^{2} .
$$

By definition of the norm (42) and of the $T_{j}, j=1,2, . ., 6$,

$$
\left\|\mathbf{T}_{1-6} \underline{u}\right\|\left\|_{\star, E}^{2} \leq 3 \sum_{j=4}^{6} u_{j}^{2}\right\| T_{j} \|_{\star, E}^{2}=3 \sum_{j=4}^{6} u_{j}^{2} \sum_{i=1}^{\mathcal{D}}\left|e_{i}\right| \int_{e_{i}}\left(\frac{d}{d s} \phi_{j}\right)^{2} .
$$

Since all the derivatives of the $\phi_{j}$ are bounded by 1 , and $\left|e_{i}\right|^{2} \leq C|E|$, we get

$$
\left\|\mathbf{T}_{1-6} \underline{u}\right\|_{\star, E}^{2} \leq C \sum_{j=4}^{6} u_{j}^{2} \sum_{i=1}^{\mathcal{D}}\left|e_{i}\right|^{2} \leq C|E| \|\left.\underline{u}\right|_{\star} ^{2} .
$$

We now observe that, for all $W_{E} \in X_{E}$, the restriction of the associated function $\mathbf{w}_{E}$ to the boundary is a piecewise polynomial function. Therefore an edge-by-edge standard inverse inequality gives

$$
\left\||| W_{E}\right\|_{E}^{2}=\sum_{i=1}^{\mathcal{N}(E)}\left|e_{i}\right| \int_{e_{i}}\left(\frac{d}{d s} \mathbf{w}_{E}(s)\right)^{2} d s \leq C|| \mathbf{w}_{E} \|_{L^{\infty}(\partial E)}^{2} .
$$

The definition of the degrees of freedom in Section 3.1, combined with (50), easily implies

$$
\left\|W_{E}:\right\|_{E}^{2} \leq C\left\|W_{E}\right\|^{2} \quad \forall W_{E} \in X_{E} .
$$

Using (28), (51) and recalling the scaling assumption (30) yields

$$
\left\|\mathbf{T}_{7-\mathcal{D}} \underline{v}\right\|_{\star, E}^{2} \leq\left\|\left|\mathbf{T}_{7-\mathcal{D}} \underline{v}\left\|_{E}^{2} \leq\left. C|| \mathbf{T}_{7-\mathcal{D}} \underline{v}\right|^{2}=C|E|\right\| \underline{v} \|^{2} .\right.\right.
$$

The result follows combining (48), (49) and (52) with lower bounds in (46):

$$
\left\|V_{E}\right\|_{*, E}^{2} \leq C|E|\left(\|\underline{u}\|_{*}^{2}+\|\underline{v}\|^{2}\right) \leq C_{1}\left(\underline{u}^{T} \tilde{\mathbf{A}}_{E}^{11} \underline{u}+\underline{v}^{T} \tilde{\mathbf{A}}_{E}^{22} \underline{v}\right)=C_{1} V_{E}^{T} \mathbf{A}_{E} V_{E} .
$$

The assertion of the lemma follows with $\alpha_{E}=C_{1}$.

The bilinear form $\mathcal{A}_{E}\left(\mathbf{v}_{E}, \mathbf{v}_{E}\right)$ is also $h$-uniformly continuous with respect to the same norm \|\|$\cdot\|\|_{\star}$. This result is shown in Lemma 5 in the Appendix. It does not affect the main results of this paper and can be skipped. Two global estimates are presented below. We give the proof only of the first one. The second one is proved in Appendix.

Theorem 1. Let $\Omega^{h}$ be a connected partition. Then the following holds.

- \|\|$\cdot \|_{*}$ is a norm on $X_{0}^{h}$.

- There exist a positive constant $\alpha$ depending only on $\alpha_{E}$, such that

$$
V^{T} \mathbf{A} V \geq \alpha\|V\|_{*}^{2} \quad \forall V \in X^{h} .
$$


Proof. Throughout the proof we will need the following definition. For $V_{E} \in X_{E}$ define

$$
\check{V}_{E}:=V_{E}-\mathbf{T}_{1-3} \mathcal{C}
$$

where $\underline{c}$ minimizes the seminorm (42) on element $E$ and is different for different elements. Then it is obvious that

$$
V_{E}^{T} \mathbf{A}_{E} V_{E}=\tilde{V}_{E}^{T} \mathbf{A}_{E} \tilde{V}_{E}
$$

Now we prove that $\|\cdot\|_{*}$ is a norm on $X_{0}^{h}$. Clearly, $\|\cdot\|_{*}$ is a seminorm. Thus, it only remains to show that for any $V \in X_{0}^{h},\|V\|_{*}=0$ implies that $V_{E}=0$. We will prove this by contradiction. Suppose $V \in X_{0}^{h}, V \neq 0$ and $\|V\|_{*}=0$. Then $\left\|V V_{E}\right\|_{*, E}=0$ for all elements $E \in \Omega^{h}$. Without loss of generality assume that $E$ is the closest element to the boundary for which $V_{E} \neq 0$ but $\left\|V_{E}\right\|_{*, E}=0$. Due to (55) this implied that

$$
V_{E}=\mathbf{T}_{1-3} \underline{c} .
$$

Since $E$ is the closest element to the boundary, it contains as least one edge along which $V$ is zero. Hence, due to (56) $V_{E}=0$. Contradiction. This proves that $\|\cdot \cdot\|_{*}$ is a norm on $X_{0}^{h}$.

Now we use Lemma 3 to show (53)

$$
\frac{V^{T} \mathbf{A} V}{\|V\|_{*}^{2}}=\frac{\sum_{E \in \Omega^{h}} \tilde{V}_{E}^{T} \mathbf{A}_{E} \tilde{V}_{E}}{\sum_{E \in \Omega^{h}}\left\|\tilde{V}_{E}\right\|_{*}^{2}} \geq \min _{E \in \Omega^{h}} \frac{\tilde{V}_{E}^{T} \mathbf{A}_{E} \tilde{V}_{E}}{\left\|\tilde{V}_{E}\right\|_{*}^{2}} \geq \min _{E \in \Omega^{h}} \alpha_{E} \equiv \alpha .
$$

This proves the theorem.

Theorem 2. Let meas $\left(\Gamma_{1}\right)>0$. Then it holds

$$
\|V\|_{\infty}^{2} \leq C \ln (2+1 / h) \mathcal{A}(\mathbf{v}, \mathbf{v}) \quad \forall V \in X^{h}
$$

where $C$ is independent of $h,\|\cdot\|_{\infty}$ indicates the maximum norm on vectors and $V$ is the vector representation of $\mathbf{v}$.

Note that the logarithmic factor in Theorem 2 is sharp and it is not an over-estimation. This factor appears essentially because the $H^{1}$ norm in two dimensions does not bound the $L^{\infty}$ norm.

\subsection{Inf-sup condition}

We only show briefly the proof of the following result.

Theorem 3. It exists a positive constant $\beta$ independent of h such that for all $S \in Q^{h}$ (and the associated piecewise constant function s) it exists $V \in X_{0}^{h}$ (and the associated piecewise regular function $\mathrm{v}_{h}$ ) such that

$$
\mathcal{B}(s, \mathbf{v}) \geq \beta\left(\sum_{E \in \Omega_{h}}|E|\left|s_{E}\right|^{2}\right)^{1 / 2}, \quad\|V\| \|_{\star} \leq 1
$$

Proof. Due to the well known infsup condition [9] of the continuous problem we have the existence of $a \widetilde{v} \in X_{0}$ such that

$$
\mathcal{B}(s, \widetilde{\mathbf{v}}) \geq \beta^{\prime}\|s\|_{\nu^{2}(\Omega)}=\beta^{\prime}\left(\sum_{E \in \Omega_{h}}|E|\left|s_{E}\right|^{2}\right)^{1 / 2}, \quad\|\widetilde{\mathbf{v}}\|_{H^{2}(\Omega)} \leq 1 .
$$


with $\beta^{\prime}>0$ and independent of $s$. We then introduce the piecewise linear function $\widetilde{\mathbf{v}}_{c}$ on the sub-mesh $S_{h}$ given by the classical Clement interpolant [16] of $\widetilde{v}$. It holds

$$
\left\|\widetilde{\mathbf{v}}_{c}\right\|_{H^{1}(\Omega)} \leq C\|\widetilde{\mathbf{v}}\|_{H^{1}(\Omega)} \leq C
$$

We then introduce $V \in X_{0}^{h}$ (and the related $\mathbf{v}$ ) defined on each element $E$ by

$$
\begin{array}{ll}
\left(V_{i}^{x}, V_{i}^{y}\right)=\widetilde{\mathbf{v}}_{c}\left(\mathbf{a}_{i}\right) & i=1, \ldots, \mathcal{N}(E), \\
V_{i}^{e}=\frac{1}{\left|e_{i}\right|} \int_{\epsilon_{i}} \tilde{\mathbf{v}}(s) \cdot \mathbf{n}_{e} d s & i=1, \ldots, \mathcal{N}(E) .
\end{array}
$$

Due to $(61)_{2}$ and the definition of $\mathcal{B}$ it is easy to check that

$$
\mathcal{B}(s, \mathbf{v})=\sum_{E \in \Omega_{h}} s_{E} \int_{e_{\mathfrak{i}}} \tilde{\mathbf{v}} \cdot \mathbf{n}_{e} d s=\sum_{E \in \Omega_{h}} s_{E} \int_{E} \operatorname{div}(\tilde{\mathbf{v}}) d x=\mathcal{B}(s, \tilde{\mathbf{v}}),
$$

which together with the first property in (59) immediately implies the first part of (58). The second bound in (58) follows with a scaling argument and recalling (60).

\section{Numerical experiments}

To measure quality of a discrete solution, we define two mesh-dependent $L^{2}$ norms

$$
\|V\|_{x}=\left[\sum_{E \in \Omega^{h}}|E| V_{E}^{T} V_{E}\right]^{1 / 2} \quad \text { and } \quad\|P\|_{Q}=\left[\sum_{E \in \Omega^{h}}|E| P_{E}^{2}\right]^{1 / 2} \text {. }
$$

To solve the saddle point problem (14), we use the iterative solvers with block diagonal preconditioners of the form

$$
\left[\begin{array}{cc}
\mathbf{H}_{0} & 0 \\
0 & \mathbf{M}
\end{array}\right]
$$

where $\mathbf{H}_{0}$ is a preconditioner for the matrix $\mathbf{A}_{0}$ and $\mathbf{M}_{0}$ is the diagonal mass matrix with areas $|E|$ on the diagonal. To achieve a mesh independent convergence of the iterative process, the matrix $\mathbf{H}_{0}$ must be spectrally equivalent to $\mathbf{A}_{(0)}$. We have verified this for small mesh resolutions and $\mathbf{H}_{0}=\mathbf{A}_{0}^{-1}$. However, we have not achieved spectral equivalence with either the algebraic multigrid [29] or the second-order accurate incomplete LU factorization [23].

\subsection{Random quadrilateral meshes}

Let $\Omega$ be a unit square and $\nu=1 / 2$. We impose the Dirichlet boundary conditions on three sides of the unit square and the Neumann boundary condition of the remaining side. These conditions are chosen such that the exact solution is

$$
\mathbf{u}(x, y)=\left[\begin{array}{l}
y^{3}+x \\
x^{3}-y
\end{array}\right], \quad p(x, y)=3 x y-.75
$$

We consider a sequence of randomly perturbed quadrilateral meshes (see Fig.2). A randomly perturbed mesh is built from a square mesh with mesh size $h=1 / n$ by relocating each interior mesh node a to a random position inside a square box. The box is centered at $\mathbf{a}$, its sides are aligned with the coordinate axis, and its size is $h / 2$.

The convergence analysis on the sequence of randomly perturbed meshes is the most challenging test for any discretization method. Figure 3 shows the second-order convergence rate for the discrete $L^{2}$ norm of the velocity error and the first-order for the discrete $L^{2}$ norm of the pressure error and the discrete $H^{1}$ norm (43) of the velocity error. 

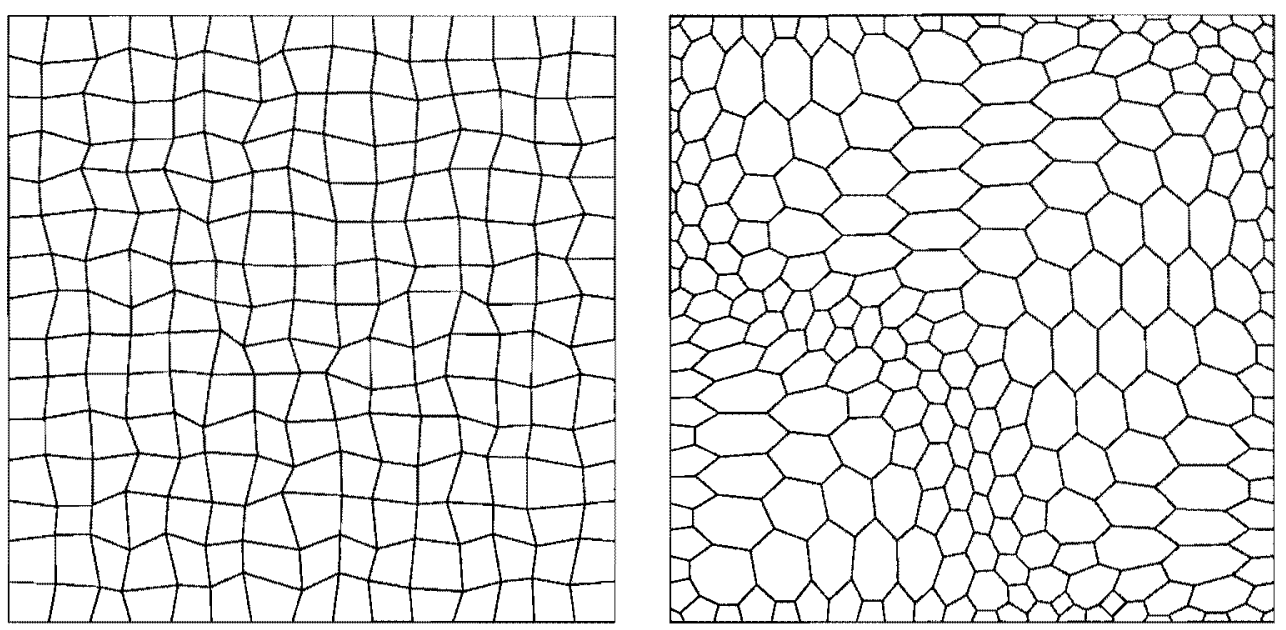

Figure 2: A sample $15 \times 15$ quadrilateral mesh with randomly perturbed vertices and a sample polygonal mesh.
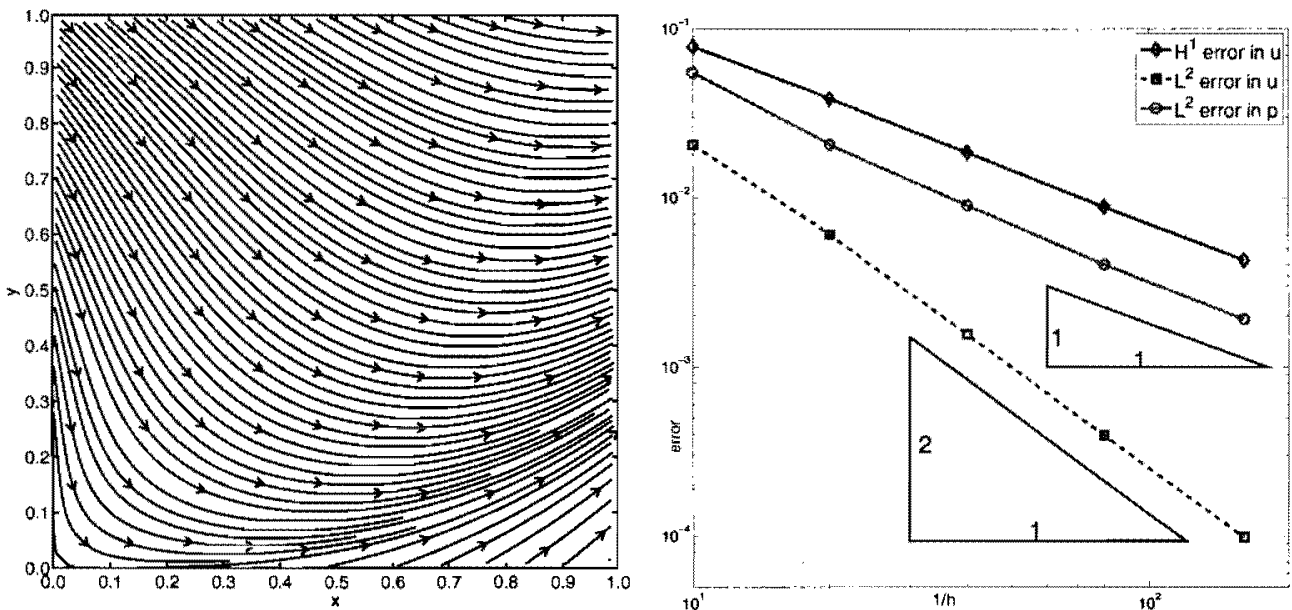

Figure 3: Left picture shows the streamlines for the discrete solution on the finest mesh. Right picture shows graphs of the mesh-dependent $L^{2}$ and $H^{1}$ norms of errors. The velocity graphs are marked by diamonds and squares (blue lines) and the pressure graph is marked by circles (red line).

\subsection{Polygonal meshes}

Let $\Omega$ be, again, a unit square and $\nu=1 / 2$. We consider the Dirichiet boundary value problem with the exact solution

$$
\mathbf{u}(x, y)=e^{x+y}\left[\begin{array}{c}
1 \\
-1
\end{array}\right], \quad p(x, y)=0
$$

Contrary to (62) this solution results in a non-zero right-hand side.

We study convergence of the method on a sequence of polygonal meshes. A polygonal mesh (see Fig. 2) is built in two steps. First, we generate the Voronoi tessellation for the set of points 
$\left(x_{i, j}, y_{i, j}\right)$ given by

$$
\begin{array}{ll}
x_{i, j}=\xi_{i}+0.1 \sin \left(2 \pi \xi_{i}\right) \sin \left(2 \pi \eta_{j}\right), & i=0, \ldots, n, \\
y_{i, j}=\eta_{j}+0.1 \sin \left(2 \pi \xi_{i}\right) \sin \left(2 \pi \eta_{j}\right), & j=0, \ldots, n,
\end{array}
$$

where $\xi_{i}=i h, \eta_{j}=j h$ and $h=1 / n$. Second, we move each interior mesh node a to the center of mass of a triangle formed by the centers of three Voronoi cells sharing a.

As shown in Fig. 4, we observe the second-order convergence rate for the discrete $L^{2}$ norm of the velocity error. Some superconvergence (higher order than 1) is observed for the discrete $H^{1}$ norm of the velocity error and the discrete $L^{2}$ norm of the pressure error. This reflects the fact that the sequence of polygonal meshes has been build using the smooth map.
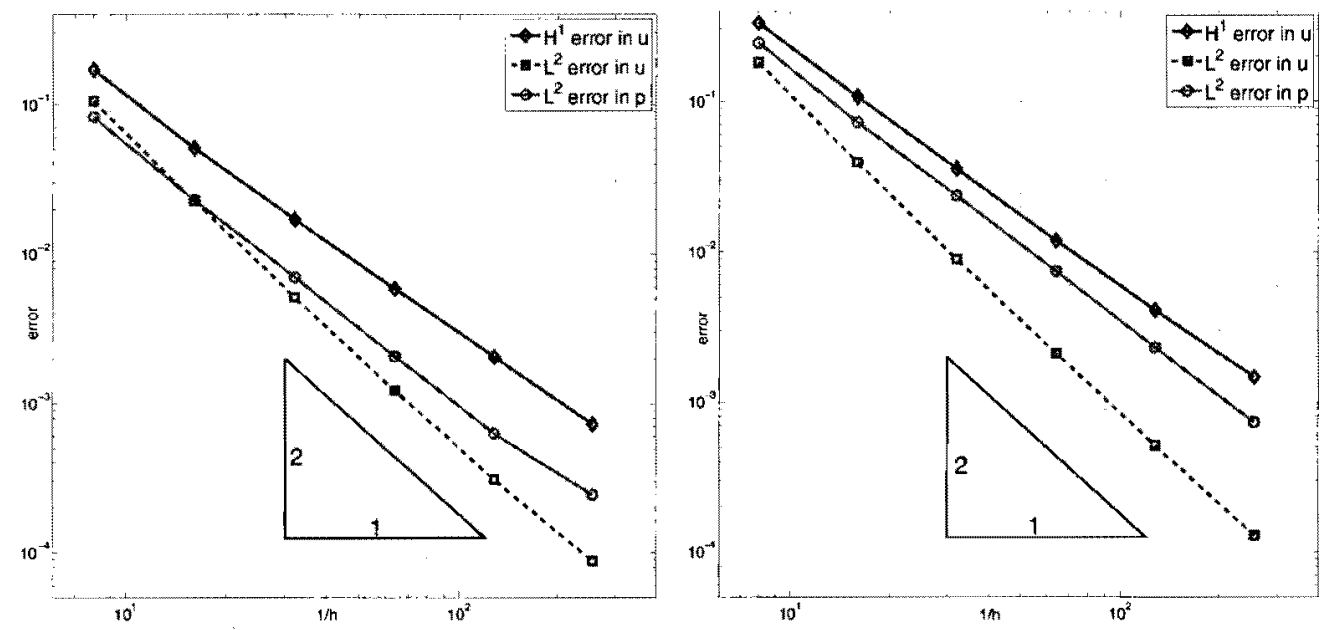

Figure 4: Graphs (left is for $\nu$ that is a scalar and right is for $\nu$ that is a tensor) of the meshdependent $L^{2}$ and $H^{1}$ norms of errors. The velocity graphs are marked by diamonds and squares (blue lines) and the pressure graphs are marked by circles (red lines).

\subsection{Polygonal meshes and tensor coefficients}

Let us consider the previous example but replace the constant viscosity $v$ by a symmetric fourthorder tensor $\nu$ satisfying (2). Let $\varepsilon=D(\mathbf{u})$. Using the reduced Voigt notation, the symmetric tensor is defined by six independent components:

$$
\left[\begin{array}{l}
\sigma_{x x} \\
\sigma_{y y} \\
\sigma_{x y}
\end{array}\right]=\left[\begin{array}{lll}
\nu_{11} & \nu_{12} & \nu_{13} \\
\nu_{12} & \nu_{22} & \nu_{23} \\
\nu_{13} & \nu_{33} & \nu_{33}
\end{array}\right]\left[\begin{array}{l}
\varepsilon_{x x} \\
\varepsilon_{y y} \\
\varepsilon_{x y}
\end{array}\right]-\left[\begin{array}{l}
p \\
p \\
0
\end{array}\right] .
$$

This problem is an intermediate step towards the displacement formulation of a linear elasticity problem. Therefore, we set $\nu_{11}=\nu_{22}=\lambda+2 \mu, \nu_{12}=\lambda$ and $\nu_{33}=4 \mu$, all other coefficients are then equal to zero. The anisotropic tensor is obtained by setting $\lambda=10$ and $\mu=1$.

The right picture in Fig. 4 shows the second-order convergence rate for the discrete $H^{1}$ norm of the velocity error. Again, slight superconvergence is observed for the other two errors. Comparing the two pictures in Fig. 4, we see that the effect of the tensor anisotropy is mild. All errors are roughly twice bigger than that for the scalar coefficient $\nu$. 


\subsection{Locally refined meshes}

Let $\Omega$ be again the unit square and $\nu=2$. We consider the Dirichlet boundary value problem with the point force $\mathbf{F}=(\delta(0.5,0.5), 0)^{T}$ in the middle of $\Omega$. The exact solution is

$$
\mathbf{u}(x, y)=\frac{1}{8 \pi}\left[\begin{array}{c}
1+\log \left(x^{2}+y^{2}\right)+\frac{2 y^{2}}{x^{2}+y^{2}} \\
\frac{-2 x y}{x^{2}+y^{2}}
\end{array}\right], \quad p(x, y)=\frac{-4 x}{x^{2}+y^{2}}
$$

We study convergence of the method on a sequence of locally refined meshes. The sequence starts with the uniform square $16 \times 16$ mesh. The singular point force is approximated by a piecewise constant function with unit integral. On each mesh in the sequence, this function equals to zero almost everywhere except in four square cells in the middle of the domain. The mesh refinement is based on a simple error indicator - sum of pressure jumps across mesh edges. The error threshold for mesh refinement is the average value of this indicator.
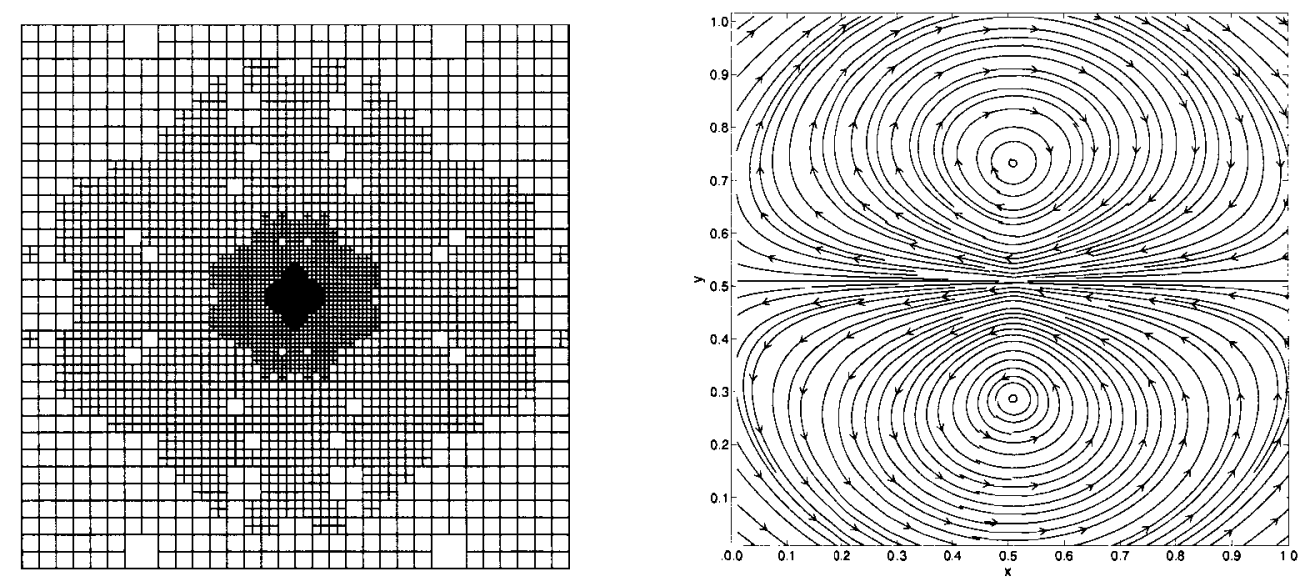

Figure 5: Left picture shows the locally refined mesh after 3 adaptive iterations. Right picture shows streamlines of the discrete solution on the most refined mesh.

Figure 5 indicates strong refinement in the domain center where the solution is singular. Since $p$ is not in $L^{2}(\Omega)$, we do not have convergence for the discrete $L^{2}$ norm of the pressure error, see Table 1. For the same reason, the discrete $H^{1}$ norm of the velocity error does not converge to zero. However, convergence rate for the discrete $L^{2}$ norm of the velocity error is 1.7 . The linear regression method has been used to estimate the error reduction rate with respect to the effective mesh size $h_{\text {eff }}=1 / \sqrt{\mathcal{N}\left(\Omega^{h}\right)}$.

\begin{tabular}{r|c|c|c}
$\mathcal{N}\left(\Omega^{h}\right)$ & $\left\|\mid U^{e x}-U\right\|_{X}$ & $\left\|U^{e x}-U\right\|_{*}$ & $\left\|P^{e x}-P\right\|_{Q}$ \\
\hline 256 & $4.47 \mathrm{e}-2$ & $6.04 \mathrm{e}-1$ & $2.47 \mathrm{e}-1$ \\
472 & $2.56 \mathrm{e}-2$ & $7.04 \mathrm{e}-1$ & $2.48 \mathrm{e}-1$ \\
928 & $1.46 \mathrm{e}-2$ & $8.06 \mathrm{e}-1$ & $2.47 \mathrm{e}-1$ \\
1924 & $8.06 \mathrm{e}-3$ & $9.01 \mathrm{e}-1$ & $2.47 \mathrm{e}-1$ \\
3868 & $4.45 \mathrm{e}-3$ & $1.02 \mathrm{e}-2$ & $2.47 \mathrm{e}-1$
\end{tabular}

Table 1: Convergence on a sequence of locally refined meshes. 


\section{Conclusion}

We have developed a mimetic finite difference (MFD) method for the Stokes problem. Contrary to a finite element method, the MFD method uses only the surface representation of discrete unknowns to build stiffness and mass matrices. Since no extension inside the mesh element is required, practical implementation of the MFD method is simple for polygonal meshes that may include degenerate and non-convex elements. The method is constructed for tensor coefficients, which will allow to apply it to a linear elasticity problem. The numerical experiments show the second-order convergence for the velocity variable and the first-order for the pressure in mesh dependent $L^{2}$ norms.

\section{Acknowledgments}

This work was carried out under the auspices of the National Nuclear Security Administration of the U.S. Department of Energy at Los Alamos National Laboratory under Contract No. DE-AC52-06NA25396 and the DOE Office of Science Advanced Scientific Computing Research (ASCR) Program in Applied Mathematics Research.

\section{A Appendix}

Lemma 4. For all $V_{E} \in X_{E}$

$$
\min _{c \in \mathbb{R}^{3}}\left\|V_{E}+\mathbf{T}_{1-3} c\right\| \leq C\left\|V_{E}\right\|_{\star, E}
$$

Proof. We start observing that $\phi_{1}$ and $\phi_{3}$ span the space of the constant vector fields on $E$. Therefore, as in the proof of the previous lemma, a simple one dimensional scaling argument on the boundary gives

$$
\min _{c_{1}, c_{3} \in \mathbb{R}}\left\|\mathbf{v}_{E}+c_{1} \phi_{1}+c_{3} \phi_{3}\right\|_{L^{\infty}(\partial E)}^{2} \leq C \sum_{i=1}^{N(E)}\left|e_{i}\right| \int_{e_{i}}\left(\frac{d}{d s} \mathbf{v}_{E}(s)\right)^{2} d s=C\left\|V_{E}\right\|_{E}^{2} .
$$

From the definition of the degrees of freedom of $X_{E}$ we now have

$$
\left\|V_{E}\right\| \leq C|| \mathbf{v}_{E} \|_{L^{\infty}(\partial E)}
$$

which combined with (65) gives

$$
\min _{c_{1}, c_{3} \in \mathbb{R}}\left\|V_{E}+c_{1} T_{1}+c_{3} T_{3}\right\| \leq C \min _{c_{1}, c_{3} \in \mathbb{R}}\left\|\mathbf{v}_{E}+c_{1} \phi_{1}+c_{3} \phi_{3}\right\|_{L_{\infty} \infty}(\partial E) \leq C\left\|V_{E}\right\| \|_{E}
$$

The result finally follows from $(66)$ and by definition

$$
\min _{C \in \mathbb{R}^{3}}\left\|V_{E}+\mathbf{T}_{1-3} \subseteq\right\| \leq C \min _{C_{2} \in \mathbb{R}^{R}}\left\|V_{E}+c_{2} T_{2}\right\|\left\|_{E}=C\right\| V_{E} \|_{\star, E}
$$

Lemma 5. It exists a positive constant $C_{E}$, depending only on the tensor $\nu$ and the geometric constants in Section 4.1.2, such that

$$
\mathcal{A}_{E}\left(\mathbf{v}_{E}, \mathbf{v}_{E}\right)=V_{E}^{T} \mathbf{A}_{E} V_{E} \leq C_{E}\left\|V_{E}\right\|_{*, E}^{2} \quad \forall V_{E} \in X_{E}
$$

Proof. Using (47) and the relations (26), (27) it follows

$$
\mathbf{R}_{E}^{T} V_{E}=\mathbf{R}_{E}^{T} \mathbf{T}_{1-6} \underline{u}+\mathbf{R}_{E}^{T} \mathbf{T}_{7-\mathcal{D}} \underline{y}=\tilde{\mathbf{A}}_{E}^{11} \underline{u} .
$$


First due to (29) and Lemma 2, then using (67), we obtain

$$
\|\underline{u}\|_{\star}^{2} \leq C|E|^{-1} \underline{u}^{T} \tilde{\mathbf{A}}_{E}^{11} \underline{u}=C|E|^{-1} \underline{u}^{T} \mathbf{R}_{E}^{T} V_{E} .
$$

A Cauchy-Schwartz inequality applied to (68), using also that the first three components of $\mathbf{R}_{E}^{T} V_{E}$ are null, gives

$$
\|\underline{u}\|_{\star}^{2} \leq C|E|^{-1}|| \underline{u}\left\|_{\star}\right\| \mathbf{R}_{E}^{T} V_{E} \|_{\star},
$$

which immediately implies

$$
\|\underline{u}\|_{*} \leq C|E|^{-1} \mid\left\{\mathbf{R}_{E}^{T} V_{E} \|\right.
$$

Due to the assumptions in Section 4.1.2 and definition (22), it is easy to check that

$$
\left\|\mathbf{R}_{E}^{T} W_{E}\right\| \leq C|E|^{1 / 2} \mid\left\|W_{E}\right\| \quad \forall W \in X_{E} .
$$

Properties (29) and (26) imply that $\mathbf{R}_{E}^{T} T_{j}=0$ for $j=1,2,3$. Therefore, applying also (71) and afterwards Lemma 4, it follows

$$
\begin{aligned}
\left\|\mathbf{R}_{E}^{T} V_{E}\right\| & =\min _{E \in \mathbb{R}^{3}}\left\|\mathbf{R}_{E}^{T}\left(V_{E}+\mathbf{T}_{1-3 c}\right)\right\| \leq C|E|^{1 / 2} \min _{c \in \mathbb{R}^{3}}\left\|V_{E}+\mathbf{T}_{1-3 c}\right\| \\
& \leq C|E|^{1 / 2} \mid\left\|V_{E}\right\|_{\star, E} .
\end{aligned}
$$

The bounds (70) and (72) give

$$
\left\|V_{E}\right\|_{\star, E} \geq \beta_{E}|E|^{1 / 2}|| \underline{u} \|_{\star},
$$

where $\beta_{E}$ is a $|E|$-uniformly positive constant.

Let $c_{1}$ and $c_{2}$ be two reals, such that $0<c_{1}+c_{2}=1$ (exact definition will follow in (76)). We now write, using (73), (47) and a triangle inequality,

$$
\begin{aligned}
\left\|V_{E}\right\|_{\star, E} & =c_{1}\left\|V_{E}\right\|_{\star, E}+c_{2}\left\|V_{E}\right\| \|_{\star, E} \\
& \geq c_{1} \beta_{E}|E|^{1 / 2}\|\underline{u}\|_{\star}+c_{2}\left\|\mathbf{T}_{1-6} \underline{u}+\mathbf{T}_{7-\mathcal{D}} \underline{v}\right\|_{\star, E} . \\
& \geq c_{1} \beta_{E}|E|^{1 / 2}\|\underline{u}\|_{\star}-c_{2}\left\|\mathbf{T}_{1-6} \underline{u}\right\|\left\|_{\star, E}+c_{2}\right\| \mathbf{T}_{7-\mathcal{D}} \underline{u} \|_{\star, E}
\end{aligned}
$$

From bound (49) and (74) we infer

$$
\left\|V_{E}\right\|\left\|_{\star, E} \geq\left(c_{1} \beta_{E}-c_{2} C_{*}\right)|E|^{1 / 2}\right\| \underline{u}\left\|_{\star}+c_{2}\right\| \mathbf{T}_{7-\mathcal{D}} \underline{u} \|_{*, E}
$$

where by $C_{*}$ we labeled the square root of the constant in (49). Making the choice

$$
c_{1}=\frac{1+C_{*}}{1+\beta_{E}+C_{*}} \quad \text { and } \quad c_{2}=\frac{\beta_{E}}{1+\beta_{E}+C_{*}},
$$

the bound (75) gives

$$
\left\|\left|V_{E}\right|\right\|_{\star, E} \geq \alpha_{E}^{\prime}\left(|E|^{1 / 2}\|\underline{u}\|_{\star}+\left\|\mid \mathbf{T}_{7-\mathcal{D}} \underline{w}\right\|_{\star, E}\right),
$$

where $\alpha_{E}^{\prime}=\frac{\beta_{E}}{1+\beta_{E}+C_{*}}$ is a $|E|$-uniform positive constant. First, due to (30), then using the orthogonality relation (28) and finally applying Lemma 4 , we get

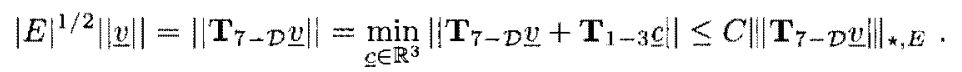

The result follows, combining (77), (78) and the equivalence (46). 
Proof of Theorem 2. Let $E$ be a generic element of $\Omega_{h}$. We call $M_{E}^{2} \subset H^{1}(E)$ the space of continuous piecewise quadratic 2-component vector functions on the local submesh $S_{h} \mid E$. Given any $V \in X^{h}$, we define $R_{E} V \in M_{E}^{2}$ as the solution of the standard problem

$$
\left\{\begin{array}{lr}
\int_{E} \nabla R_{E} V \cdot \nabla \mathbf{w}_{E}=0 & \forall \mathbf{w}_{E} \in M_{E}^{2} \cap H_{0}^{1}(E) \\
R_{E} V=\mathbf{v}_{E} & \text { on } \partial E
\end{array}\right.
$$

where we recall that $\mathbf{v}_{E}=\left.\mathbf{v}\right|_{E}$ is known explicitly and is piecewise quadratic on $\partial E$, as shown in Section 3.2. Note that the second condition fully determines $R_{E} V$ on the boundary of $E$. Therefore a well known result on discrete harmonic functions, see for instance [32], gives

$$
\left|R_{E} V\right|_{H^{1}(E)} \leq C\left|\mathbf{v}_{E}\right|_{H^{1 / 2}(O E)}
$$

which recalling (41) and using a standard scaling yields

$$
\left|R_{E} V\right|_{H^{x}(E)} \leq C\left\|V_{E}\right\|_{E}
$$

where as usual $V_{E}$ is the vector representation of $v_{E}$.

Let now $R_{h} V$ be the global piecewise quadratic vector function on the triangular submesh $S_{h}$ such that $\left.R_{h} V\right|_{E}=R_{E} V$. Recalling the definition of the degrees of freedom of $X^{h}$, it is easy to check that

$$
\|V\|_{\infty}^{2} \leq C\left\|R_{h} V\right\|_{L^{\infty}(\Omega)}^{2}
$$

Combining a classical result, see for instance [7], with the above bound gives

$$
\|V\|_{\infty}^{2} \leq C \ln (2+1 / h)^{1 / 2}\left\|R_{h} V\right\|_{H^{1}(\Omega)}^{2}
$$

Due to our assumption meas $\left(\Gamma_{1}\right)>0$ we can apply the Korn's inequality, see [15], which using (81) yields

$$
\|V\|_{\infty}^{2} \leq C \ln (2+1 / h)\left\|D\left(R_{h} V\right)\right\|_{L^{2}(\Omega)}^{2} .
$$

Following the notation already introduced in (19), we indicate with $\phi_{2}^{E}$ the vector function on $E$ which spans the rotations around the barycenter, while we indicate with $T_{2}^{E}$ its vector representation in $X_{E}$. Note that, since $\phi_{2}^{E}$ is linear on $E$ and the operator $R_{E}$ "preserves linear functions" by definition, $R_{E} T_{2}^{E}=\phi_{2}^{E}$. We then have

$$
\begin{aligned}
\left\|D\left(R_{h} V\right)\right\|_{L^{2}(\Omega)}^{2} & =\sum_{E \in \Omega_{h}}\left\|D\left(R_{E} V\right)\right\|_{L^{2}(E)}^{2}=\sum_{E \in \Omega_{h}} \min _{c \in \mathbb{R}}\left\|D\left(R_{E} V+c \phi_{2}^{E}\right)\right\|_{L^{2}(E)}^{2} \\
& \leq C \sum_{E \in \Omega_{h}} \min _{c \in \mathbb{R}}\left\|\nabla\left(R_{E} V+c \phi_{2}^{E}\right)\right\|_{L^{2}(E)}^{2} \\
& =C \sum_{E \in \Omega_{h}} \min _{c \in \mathbb{R}}\left\|\nabla R_{E}\left(V+c T_{2}^{E}\right)\right\|_{L^{2}(E)}^{2}
\end{aligned}
$$

Using bound (80) and definition (42) it follows

$$
\min _{c \in \mathbb{R}}\left\|\nabla R_{E}\left(V+c T_{2}^{E}\right)\right\|_{L^{2}(E)}^{2} \leq C \min _{c \in \mathbb{R}}\left\|V+c T_{2}^{E}\right\|\left\|_{E}^{2}=C\right\| V \|_{*, E}^{2}
$$

The result follows combining (82), (83), (84) with Proposition 3.

\section{References}

[1] J.E. Aarnes, S. Krogstad, and K.-A. Lie. Multiscale mixed/mimetic methods on cornerpoint grids. Comp. Geosciences, 2007. to appear in Special Issue on Multiscale Methods. 
[2] L. Beirão da Veiga. A mimetic finite difference method for linear elasticity. Technical report, Preprint IMATI-PV, 2008. submitted.

[3] L. Beirão da Veiga. A residual based error estimator for the mimetic finite difference method. Numer. Math., 108(3):387-406, 2008.

[4] L. Beirão da Veiga and G. Manzini. An a-posteriori error estimator for the mimetic finite difference approximation of elliptic problems with general diffusion tensor. Int. $J$. for Numer. Meth. in Engnr., 2008. in press, DOI:10.1002/nme.2377.

[5] L. Beirão da Veiga and G. Manzini. The high-order formulation of the mimetic finite difference method. SIAM J. Sci. Comput., 2008. in press.

[6] L. Beirao da Veiga, K. Lipnikov, and G. Manzini. Convergence analysis of the high-order mimetic finite difference method. Technical report, Preprint IMATI-PV 14PV08/13/0, 2008. submitted.

[7] J. H. Bramble and J. Xu. Some estimates for a weighted $l^{2}$ projection. Math. of Comp., 56:463-476, 1991.

[8] F. Brezzi, A. Buffa, and K. Lipnikov. Mimetic finite differences for elliptic problems. in preparation, 2007.

[9] F. Brezzi and M. Fortin. Mized and hybrid finite element methods, Springer-Verlag, New York, 1991.

[10] F. Brezzi, K. Lipnikov, and M. Shashkov. Convergence of the mimetic finite difference method for diffusion problems on polyhedral meshes. SIAM J. Numer. Anal., 43(5):18721896, 2005.

[11] F. Brezzi, K. Lipnikov, M Shashkov, and V. Simoncini. A new discretization methodology for diffusion problems on generalized polyhedral meshes. Comput. Methods Appl. Mech. Engrg., 196:3682-3692, 2007.

[12] F. Brezzi, K. Lipnikov, and V. Simoncini. A family of mimetic finite difference methods on polygonal and polyhedral meshes. Math. Models Methods Appl. Sci., 15(10):1533-1551, 2005.

[13] J. Campbell and M. Shashkov. A tensor artificial viscosity using a mimetic finite difference algorithm. J. Comput. Phys., 172:739-765, 2001.

[14] A. Cangiani and G. Manzini. Flux reconstruction and pressure post-processing in mimetic finite difference methods. Comput. Methods Appl. Mech. Engrg., 197/9-12:933-945, 2008.

[15] P.G. Ciarlet. Mathematical Elasticity: Volume I: Three-Dimensional Elasticity. NorthHolland, Amsterdam, 1994.

[16] Ph. Clément. Approximation by finite element functions using local regularization. RAIRO Analyse Numérique, $9(\mathrm{R}-2): 77-84,1975$.

[17] L. Codecasa and F. Trevisan. Constitutive equations for discrete electromagnetic problems over polyhedral grids. J. Comput. Phys., 225(2):1894-1918, 2007.

[18] P. Dvorak. New element lops time off CFD simulations. Mashine Design, 78(169):154-155, 2006.

[19] R. Eymard, T. Gallouët, and R. Herbin. A new finite volume scheme for anisotropic diffusion problems on general grids: convergence analysis. C. R. Math. Acad. Sci. Paris, 344(6):403-406, 2007. 
[20] V. Gradinaru and R. Hiptmair. Whitney elements on pyramids. Electron. Trans. Numer. Anal., 8:154-168, 1999.

[21] V. Gyrya and K. Lipnikov. High-order mimetic finite difference method for diffusion problems on polygonal meshes. J. Comput. Phys., 227:8841-8854, 2008.

[22] J. Hyman and M. Shashkov. Mimetic discretizations for Maxwell's equations and the equations of magnetic diffusion. Progress in Electromagnetic Research, 32:89-121, 2001.

[23] I. E. Kaporin. High quality preconditioning of a general symmetric positive definite matrix based on its $u^{t} u+u^{t} r+r^{t} u$-decomposition. Numer. Linear Algebra Appl., 5(6):483-509, NOV-DEC 1998.

[24] Yu. Kuznetsov and S. Repin. New mixed finite element method on polygonal and polyhedral meshes. Russ. J. Numer. Anal. Math. Modelling, 18(3):261-278, 2003.

[25] S. L. Lyons, R. R. Parashkevov, and X. H. Wu. A family of $H^{1}$-conforming finite element spaces for calculations on 3D grids with pinch-outs. Numer. Linear Algebra Appl., $13(9): 789-799,2006$

[26] L. Margolin, M. Shashkov, and P. Smolarkiewicz. A discrete operator calculus for finite difference approximations. Comput. Meth. Appl. Mech. Engrg., 187:365-383, 2000.

[27] J.B. Perot and V. Subramanian. Higher-order mimetic methods for unstructured meshes. J. Comput. Phys., 219(1):68 85, 2006.

[28] M. Shashkov and S. Steinberg. Solving diffusion equations with rough coefficients in rough grids. J. Comput. Phys., 129:383-405, 1996.

[29] K. Stüben. Algebraic multigrid (AMG): experiences and comparisons. Appl. Math. Comput., $13: 419-452,1983$.

[30] A. Tabarraei and N. Sukumar. Application of polygonal finite elements in linear elasticity. Int. J. Comput. Methods, 3(4):503-520, 2006.

[31] A. Tabarraei and N. Sukumar. Extended finite element method on polygonal and quadtree meshes. Comput. Methods Appl. Mech. Engrg., 197(5):425-438, 2007.

[32] A. Toselli and O. Widlund. Domain Decomposition Methods - Algorithms and Theory. Springer-Verlag, Berlin Heidelberg, 2005. 